\title{
Nanocrystalline Silicon Clusters For The Imaging Of Dynamic Cellular Processes.
}

\author{
Pamela Vallejo ${ }^{*}$, Adaris Rodriguez ${ }^{* *}$, Luis F Fonseca ${ }^{*}$, Eduardo Rosa-Molinar ${ }^{*}$, Yury Posada ${ }^{*}$ \\ * Department of Physics, Faculty of Natural Sciences, University of Puerto Rico, P. O. Box 23343, \\ San Juan, Puerto Rico 00931-3343. \\ ** Julio García Díaz Center for Investigations in Biology University of Puerto Rico, P. O. Box \\ 21809, San Juan, Puerto Rico 00931-1809.
}

We report on the synthesis procedures and stability properties of nanocrystalline silicon clusters implanted on living tissue. We explain the preparation of the nanoclusters, their morphological and photoluminescence (PL) properties and the stability of these properties once implanted in biological material. We finally present our conclusions about the applicability of such nanostructures for imaging of dynamic cellular processes.

Si nanoparticles were initially synthesized following the top-down protocol used for the preparation of Porous Silicon ${ }^{1}$ (PSi). Crystalline Si wafers with resistivities between $0.5 \Omega \mathrm{cm}$ and $1.5 \Omega \mathrm{cm}$ were electrochemically etched in a Teflon cell and with an electrolytic solution made with $25 \% \mathrm{HF}, 50 \%$ $\mathrm{H}_{2} \mathrm{O}$ and $25 \%$ Ethanol (per volume). A $50 \mathrm{~mA} / \mathrm{cm}^{2}$ electrical current density was applied between the anode (the $\mathrm{Si}$ wafer with an $\mathrm{Al}$ ohmic contact at the bottom) and the cathode (Platinum electrode) The anodization time was 50 minutes. We produced PSi films with thicknesses of the order of $200 \mu \mathrm{m}$ and $0.5 \mathrm{~cm}^{2}$ in area. By the application of a high voltage pulse we could disengage the porous films from the Si wafers. The so resulting free-standing porous films were then washed in Ethanol and dried using the critical point $\operatorname{method}^{2}$. Several films so prepared were finally immersed in Ethanol and sonicated in an ultrasonic bath during 30 minutes, until a suspension of Si nanoclusters were obtained. Figure 1 shows a TEM image of a collection of Si nanoclusters obtained by this method. The figure shows a broad clusters' size distribution. A reduction in size dispersion is obtained by decantation and centrifugation.

Figure 2 shows the normalized PL spectra of: (a) the dried PSi film previous to sonication and (b) Si nanoclusters in ethanoic suspension after sonication. It is worth to mention that PL is quenched by Ethanol in fresh sonicated nanoclusters but it is recovered by keeping the clusters suspended in ethanol for more that 12 hours. It has been explained ${ }^{3}$ that for Si nanoparticles with sizes between $\sim 1 \mathrm{~nm}$ and $\sim 3.5 \mathrm{~nm}$, the quasi-indirect gap nature of the electronic transitions responsible for the PL makes the PL quantum yield of such nanostructures is strongly dependent on any change in their surface passivation conditions. Si nanoparticles surrounded by its oxide show intense and stable PL as in the case of $n-\mathrm{Si} / \mathrm{SiO}_{2}$ composites. The above-mentioned aging step is considered responsible of the formation of an oxide layer that improves the PL intensity and stability by isolating the Si cores from the external environment. The improved PL stability of such clusters benefits their applicability for marking purposes and becomes a key factor for the use of our nanostructures when implanted in biological tissue. Figure 3 shows the image of one nanocluster from an aged suspension. Each cluster is made of several to many nanoparticles. Raman spectroscopy of the dried PSi films here described is consistent with a size distribution of silicon nanocrystalline cores with an average diameter of $\sim 2.7 \mathrm{~nm}^{4}$. The analysis of the optical absorption spectrum of the nanoclusters showed that they can be effectively stimulated by using light sources with wavelengths below $500 \mathrm{~nm}$. 

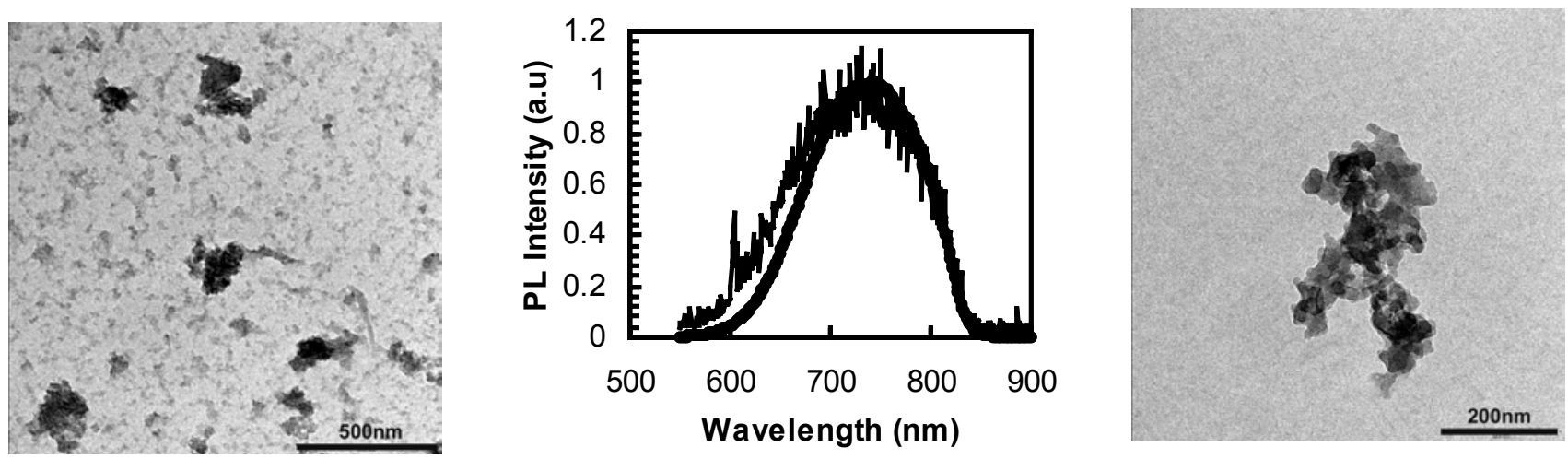

Figure 1. TEM image of Si nanoclusters from sonicated PSi.

\section{Spinal Cord \\ Nanocrystalline 7 . \\ Silicon Clusters}

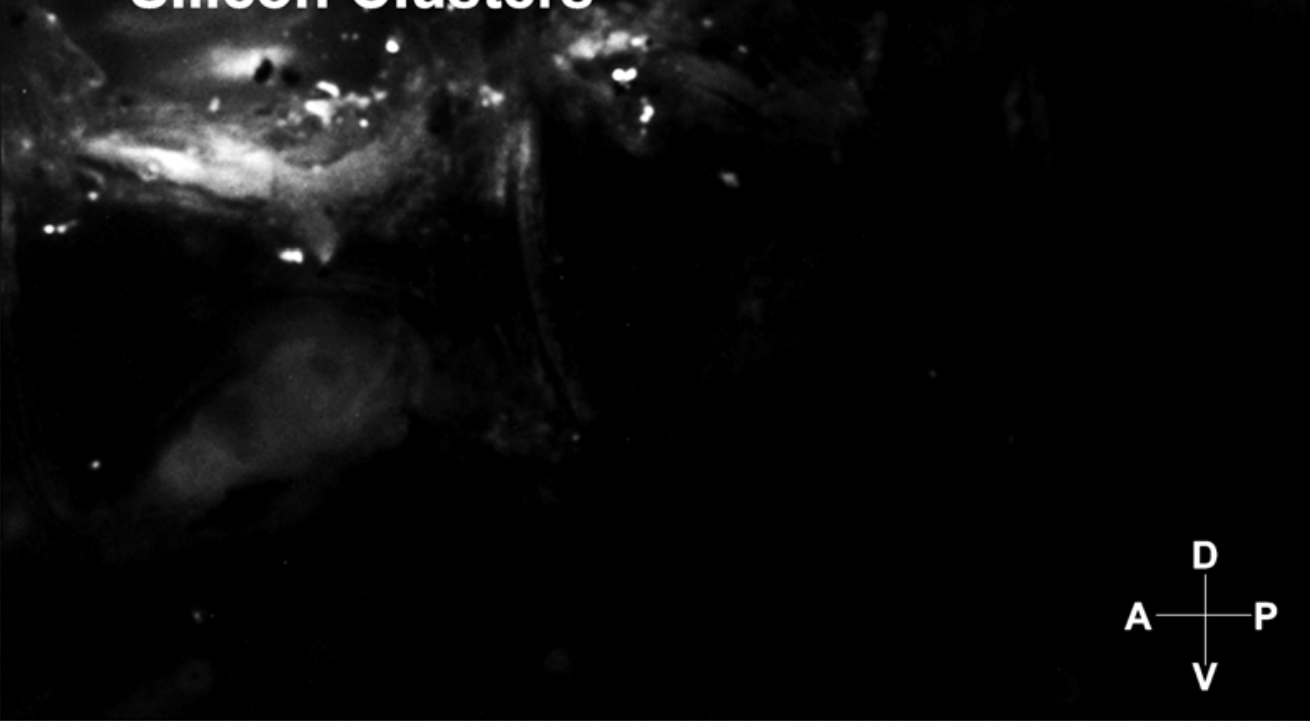

Figure 4. The implantation of the silicon nanoclusters in the living tissue was carried out by impregnating Polyethylene tubes of $13 \mathrm{~mm}$ in length and with $2 \mathrm{~mm}$ inner diameter with the nanoclusters by vacuum drying of the Ethanoic suspension and using the gene gun technique.

\section{REFERENCES}

1. L.T. Canham, Appl. Phys. Lett. 57, 1046 (1990).

2. L. T. Canham, A.G. Gullis, C. Pickering, O. D. Dosser, T. I. Cox, T. P. Lynch, Nature 368, 133 (1994).

3. M.V. Wolkin, J. Jorne, P.M. Fauchet, G. Allan, and C. Delerue, Phys. Rev. Lett. 82, 197 (1999).

4. L. F. Fonseca, O. Resto, R. Katiyar, S. Gupta, S.Z. Weisz, Y. Goldstein, A. Many and J. Shapir. 24 ICPS Proc., David Gershoni editor. World Scientific (1999). 